\title{
Development of a colonoscopy add-on device for improvement of the intubation process
}

This article was published in the following Dove Press journal:

Medical Devices: Evidence and Research

15 December 2011

Number of times this article has been viewed

\section{Jonathan D Litten' \\ JungHun $\mathrm{Choi}^{2}$ \\ David Drozek ${ }^{3}$}

'Department of Mechanical Engineering; ${ }^{2}$ Department of Mechanical Engineering and Biomedical Engineering Program; ${ }^{3}$ College of Osteopathic Medicine, Department of Specialty Medicine, Ohio University, Athens, OH, USA

Correspondence: JungHun Choi 259 Stocker Center, Department of Mechanical Engineering, Ohio University, Athens, OH 4570I, USA

Tel +l 7405932739

Fax + I 7405930476

Email choijl@ohio.edu

\begin{abstract}
A colonoscopy add-on device has been developed to reduce intubation time without modification of the current colonoscope and peripheral devices. One of the main purposes of the system is to minimize trauma caused by the distal tip of the colonoscope. The detachable sensory fixture at the end of the distal tip measures the distance between the distal tip and the colon wall in three directions, and the actuation system attached at the base of the colonoscope controls the distal tip by rotating two dial knobs. The device controls the distal tip to minimize contact between the distal tip and the colon wall, and the distal tip ideally points out the next possible lumen. A compatibility test of the infrared sensory system was carried out, and the design of the actuation system was accomplished. The system is integrated and controlled by a microprocessor. The device was tested in a silicon colon and porcine intestine. The results showed that a colonoscopist successfully reached the cecum with the aid of the colonoscopy add-on device without significant contact between the colon wall and the distal tip. The colonoscopy aid device was very helpful for the novice colonoscopist.
\end{abstract}

Keywords: colonoscope, infrared sensors, intubation, trauma, colonoscopy training model

\section{Introduction}

Prior to the invention of the colonoscope, most diagnosis of afflictions of the gastrointestinal tract was performed with barium radiology. This procedure involved using a barium enema to fill the large intestine and taking $\mathrm{x}$-rays. Although this method gives a black and white picture of the colon, it is not as accurate or as thorough as a direct inspection. As a response to this, the fully flexible and maneuverable endoscope was invented in the late 1960s. This device was improved in the 1970 s to allow the surgeon to remove abnormal features, such as polyps, for biopsy. ${ }^{1}$ Endoscopy is a procedure in which an endoscope is inserted into the body to examine the inside of a hollow organ. In the case of colonoscopy, the colonoscope is inserted into the anus of the patient and maneuvered to the end of the colon.

A typical colonoscope consists of the following elements: control head, instrument shaft, deflectable tip, biopsy forceps, light source, air/water supply, and the connecting "umbilical cord." The scope is maneuvered through the colon by use of the tip controls on the control head and the deflectable tip. When the colonoscopist turns the tip controls, the bending section at the end of the colonoscope moves to traverse through bends or narrow passages in the colon. This movement is accomplished by pull wires attached to the distal tip of the colonoscope that run down the length of the scope to the tip control knobs. The distal tip contains four channels necessary for completion of the procedure. The light channel provides illumination so that 
the surgeon can see the colon walls through the lens. The air/water jet channel supplies air to inflate the colon for better examination and squirts water to clean the lens if necessary. The biopsy/suction channel allows the insertion of biopsy tools to take samples of abnormal features in the colon. It also allows for the suctioning of fluid or polyp samples for further examination and testing. Generally, a more experienced colonoscopist should be able to reach the cecum with greater success than a less experienced colonoscopist. As a result, the cecum is reached with a colonoscope in only $90 \%$ of patients. $^{2}$ In addition to difficulty completing the procedure, colonoscopy can cause damage to the patient, such as colonic perforation and overdistention of the colonic walls. ${ }^{3}$

Although conventional colonoscopy has been established as the gold standard for screening the colon, its drawbacks have led researchers to develop improvements to colonoscopes as well as new ways of detecting colon cancer and other diseases. Nonsurgical methods offer a less invasive procedure than conventional colonoscopy. Variable stiffness colonoscopes and shaft guidance devices help solve the problem of loop formation. Robotic endoscopes are designed to travel through the colon automatically.

\section{Nonsurgical screening methods}

Because of the proliferation of colonoscopy as the main method for screening for gastrointestinal disease, several less invasive technologies have been introduced in an attempt to reduce patient discomfort and improve detection of disease. One of these technologies is virtual colonoscopy, which includes computed tomographic colonography and magnetic resonance colonography. Although virtual colonoscopy does not require actual surgery, in many cases the patient must still undergo bowel preparation for the procedure, which many patients consider the most uncomfortable aspect of colonoscopy. ${ }^{4}$ Also, virtual colonoscopy is effective only at identifying polyps greater than 6-10 $\mathrm{mm}$ in size. ${ }^{4-8}$ Another nonsurgical screening method for disease of the colon is capsule endoscopy. Through several studies it has been determined that capsule endoscopy has a low diagnostic accuracy for identifying polyps and usually requires a subsequent method of screening, such as colonoscopy. ${ }^{9-11}$

\section{Variable stiffness colonoscopes}

Loop formation in the colon can often cause difficulty in completing colonoscopy. These loops form because of the tradeoff between the stiffness and flexibility of the colonoscope. ${ }^{12}$ Variable stiffness colonoscopes (VSCs) have been developed that combine these two desired characteristics.
They have a dial on the control head that controls the stiffness of the colonoscope as desired. VSCs have been shown to achieve cecal intubation faster and to reduce the amount of pain experienced by the patient. ${ }^{13-16}$

\section{Shaft guidance}

In addition to VSCs, researchers have developed shaft guidance devices to prevent the formation of loops. These devices consist of an overtube to encircle the colonoscope. The rigidity of the device can be changed in order to absorb the force of the colonoscope while advancing it. One such device has been proposed by Raju et a $1{ }^{17}$ and uses a multilink skeleton. It has a squeeze handle that can control the stiffness of the device when needed. Yagi et al ${ }^{18}$ developed a similar device that uses an on-off switch and air pressure to control the rigidity of the sheath. The Vacu-SL, developed by Loeve et al, ${ }^{19}$ uses a vacuum pump to remove air from a foil tube filled with small particles.

\section{Computer-assisted colonoscopy}

Although some researchers have sought to solve difficulties with colonoscopy by creating new devices to work in conjunction with conventional colonoscopes, other researchers have developed new types of scopes. Eickhoff et $\mathrm{al}^{20}$ developed the NeoGuide Endoscopy System, which is similar in size and shape to conventional colonoscopes but contains 16 identical segments that are actuated independently. The NeoGuide Endoscopy System contains sensors and a controller that, as the scope is advanced through the colon, stores the information of the scope configuration. Through testing of ten patients, the NeoGuide system was shown to be effective in reaching the cecum.

\section{Earthworm-based robotic endoscopes}

In order to eliminate the need for a conventional colonoscope, several researchers have developed robotic devices that mimic the locomotion of an earthworm to navigate through the colon. One of these devices is called the EndoCrawler, developed by $\mathrm{Ng}$ et al. ${ }^{3}$ Tests performed on a live adult pig showed that the EndoCrawler was able to advance approximately $30 \mathrm{~cm}$ into the colon. Kumar et $\mathrm{al}^{21}$ proposed a similar device that uses the same earthworm locomotion principles but is used independently of a traditional colonoscope. Pressurized air propels two units that grip to the colon walls, thereby propelling the device through the bowel. Zuo et $\mathrm{al}^{22}$ developed a microcreeping robot that also acts independently of a traditional colonoscope and mimics the locomotion of an earthworm. Tests performed in a rubber pipeline showed that the robot 
can propel itself forward and backwards. Ye et $\mathrm{al}^{23}$ developed a system very similar to this one using wireless power transfer rather than wired connections. Tests showed that it was able to navigate through the intestine of a pig.

\section{Research objectives}

These studies detail different attempts to improve screening for colon cancer and other diseases. The purpose of this research is to investigate the design and preliminary testing of a new, unique device to screen for colon cancer and other diseases. The ultimate goals of the device are to accurately diagnose abnormalities, safely and effectively achieve cecal intubation, and be relatively low cost. Mechanical trauma caused by the distal tip of the colonoscope can be reduced. This design will consist of an attachable sensory system, an attachable and detachable actuation system, and a microprocessor to control the motion of the colonoscope. The sensory system will detect the position of the colonoscope by reading the distances to the colon wall. Then, the actuators will move the colonoscope tip to the center of the colon lumen. After the distal tip reaches the cecum, the actuation part can be detached for the normal colonoscopy procedure.

\section{Methods}

An inverse solution is mathematically developed to place the distal tip of the colonoscope with the infrared sensors and two direct current (DC) servo motors. The radial cross-section of the colon is approximated with an uncertainty. Instead of using the closed-form solutions developed from the inverse solution, a look-up table is generated for fast hardware control. For the hardware parts, the sensory system contains three pairs of infrared sensors positioned $120^{\circ}$ to each other to measure the distance between the colon wall and the distal tip of the colonoscope. The signal wires from the sensory fixture to the base of the colonoscope go through the biopsy channel and can be removed after the intubation process. Two DC servo motors are coupled to control the dial knobs (up-down and left-right) at the base of the colonoscope. The actuation part can be easily removable after intubation, and a colonoscopist can complete the normal retraction procedure without the aid of the device. The sensory and actuation parts are integrated with a microprocessor to implement the closedloop control system. The details of the control algorithm and hardware parts are described in the following subsections.

\section{Inverse solution}

The cross-section of the colon can be simplified as a circle with uncertainty, which is shown in Figure 1.

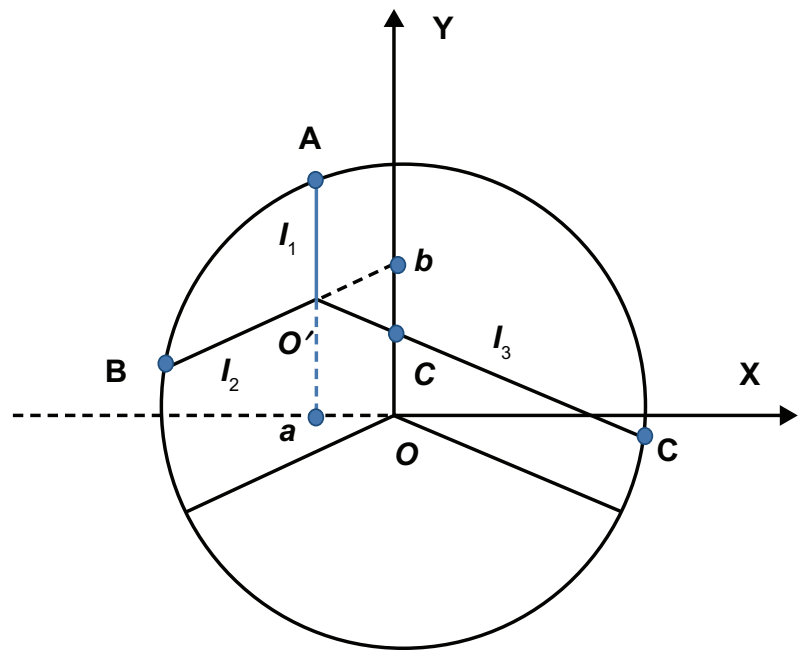

Figure I Simplified cross-section of the colon to determine control logic. ${ }^{24}$

$$
\begin{gathered}
x_{A}=a \\
y_{B}=\frac{\sqrt{3}}{3} x_{\mathrm{B}}+b \\
y_{C}=-\frac{\sqrt{3}}{3} x_{C}-c
\end{gathered}
$$

where variables $a, b$, and $c$ are the points of intersection with the $x$ - and y-axes. The slopes in Equations (2) and (3) were determined by the geometric relationship between the sensors. The sensors are positioned $120^{\circ}$ from each other, so when the sensor reading $l_{1}$ is positioned at the top, the lines overlapping line segments $l_{2}$ and $l_{3}$ have slopes shown in Equations (2) and (3) by trigonometry. The variables $a, b$, and $c$ must be found before Equations (1), (2), and (3) can be solved to find the position of the distal tip. They can be solved by using the known values of $l_{1}, l_{2}$, and $l_{3}$ from the sensors.

Equation (4) describes the shape of the simplified colon:

$$
x^{2}+y^{2}=r^{2}
$$

where $r$ is the radius of the simplified colon. The location of point $A$ in respect of point $o$ can be found by combining Equations (1) and (4) to yield Equation (5):

$$
y_{A}=\sqrt{r^{2}-a^{2}}
$$

where the $\mathrm{x}$ position, $x_{A}$, is described by Equation (1). Similarly, the location of point $B$ in respect of point $o$ can be found by combining Equations (2) and (4):

$$
x_{B^{2}}+\left(\frac{\sqrt{3}}{3} x_{B}^{2}+b\right)^{2}=r^{2}
$$




$$
2 x_{B}^{2}+\sqrt{3} b x_{B}+\frac{3}{2}\left(b^{2}-r^{2}\right)=0
$$

This equation can be solved using the quadratic formula to yield Equation (6):

$$
x_{B}=-\frac{1}{4} \sqrt{3}\left(b+\sqrt{-3 b^{2}+4 r^{2}}\right)
$$

Equation (6) can be plugged into Equation (2) to yield Equation (7):

$$
y_{B}=-\frac{1}{4}\left(3 b-\sqrt{-3 b^{2}+4 r^{2}}\right)
$$

The location of point $C$ in respect of point $o$ can be found in the same way as point $B$ using Equations (3) and (4) to yield Equations (8) and (9):

$$
\begin{gathered}
x_{C}=\frac{1}{4} \sqrt{3}\left(c+\sqrt{-3 c^{2}+4 r^{2}}\right) \\
y_{C}=\frac{1}{4}\left(3 c-\sqrt{-3 c^{2}+4 r^{2}}\right)
\end{gathered}
$$

Knowing the locations of $A, B$, and $C$, the distance formula can be used to solve for the length of line segments $l_{1}$, $l_{2}$, and $l_{3}$ in terms of $a, b, c$, and $r:^{24}$

$$
\begin{gathered}
l_{1}=\sqrt{r^{2}-a^{2}}-\frac{b+c}{2} \\
l_{2}=\sqrt{\left[-\frac{1}{4} \sqrt{3}\left(b \pm \sqrt{-3 b^{2}+4 r^{2}}\right)-a\right]^{2}+\left[-\frac{1}{4}\left(3 b \pm \sqrt{-3 b^{2}+4 r^{2}}\right)-\frac{b+c}{2}\right]^{2}} \\
l_{3}=\sqrt{\left[\frac{1}{4} \sqrt{3}\left(c \pm \sqrt{-3 c^{2}+4 r^{2}}\right)-a\right]^{2}+\left[\frac{1}{4}\left(3 c \pm \sqrt{-3 c^{2}+4 r^{2}}\right)-\frac{b+c}{2}\right]^{2}}
\end{gathered}
$$

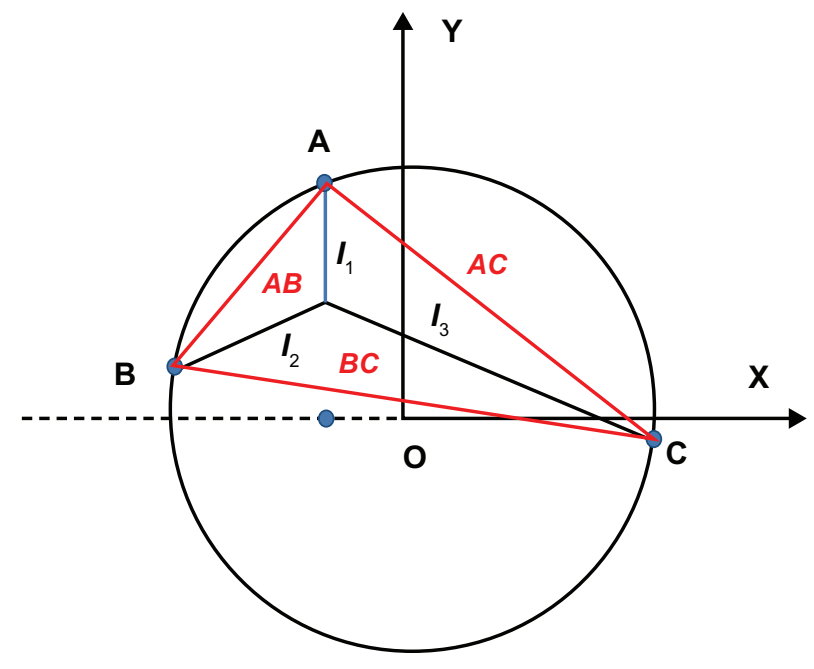

Figure 2 Altered cross-section of colon to solve for radius.

where $A B, B C$, and $A C$ are the sides of the triangle. These side lengths can be determined using the sensor readings and the angles between each sensor as shown in Figure 4.2.

Triangle $\mathrm{ABC}$ can be broken down into three smaller triangles, $\mathrm{ABO}^{\prime}, \mathrm{BCO}^{\prime}$, and $\mathrm{ACO}^{\prime}$. Each of these triangles consists of two known sensor line segments and one unknown triangle side. To determine the unknown side lengths, the law of cosines can be used:

$$
\overline{A B^{2}}=l_{1}^{2}+l_{2}^{2}-2 l_{1} l_{2} \cos \theta
$$

The radius of the colon must be known in order to solve these equations. However, the colon radius is constantly changing. Therefore, one cannot simply choose a single value for $r$; it must be solved.

Figure 2 shows the triangle $\mathrm{ABC}$, which is circumscribed by the simplified cross-section of the colon. This triangle is formed by connecting the points $A, B$, and $C$, which are the intersections of the sensor line segments $l_{1}, l_{2}$, and $l_{3}$ with the colon cross-section. The radius of the cross-section can be described by Equation (13): where $\theta$ is the angle opposite the unknown side. Because the sensors are placed evenly around the sensor fixture, the angle will always be $120^{\circ}$. Solving Equation (14) for the unknown side lengths yields Equations (15)-(17):

$$
\begin{aligned}
& \overline{A B}=\sqrt{l_{1}^{2}+l_{2}^{2}-l_{1} l_{2} \cos 120} \\
& \overline{B C}=\sqrt{l_{2}^{2}+l_{3}^{2}-l_{2} l_{3} \cos 120}
\end{aligned}
$$

$r=\frac{(\overline{A B})(\overline{B C})(\overline{A C})}{\sqrt{(\overline{A B}+\overline{B C}+\overline{A C})(-\overline{A B}+\overline{B C}+\overline{A C})(\overline{A B}-\overline{B C}+\overline{A C})(\overline{A B}+\overline{B C}-\overline{A C})}}$ 


$$
\overline{A C}=\sqrt{l_{1}^{2}+l_{3}^{2}-l_{1} l_{3} \cos 120}
$$

After solving these equations, the side lengths can be inserted into Equation (13) to solve the colon radius.

\section{Numerical solution tabulation}

Instead of using the known values of $l_{1}, l_{2}$, and $l_{3}$ from the sensors, it can be assumed that the position of the distal tip is known in relation to the center of the colon lumen. Equations (2) and (3) can be rearranged to yield Equations (13) and (14):

$$
\begin{aligned}
& b=y_{o^{\prime}}-\frac{\sqrt{3}}{3} x_{o^{\prime}} \\
& c=y_{o^{\prime}}+\frac{\sqrt{3}}{3} x_{o^{\prime}}
\end{aligned}
$$

The subscripts of $B$ and $C$ are replaced with $O^{\prime}$ because the position of the distal tip is known. By combining Equations (1), (18), and (19) with Equations (10)-(12), the distances from the sensors to the colon wall $\left(l_{\mathrm{I}}, l_{2}\right.$, and $\left.l_{3}\right)$ can be found. This process can be repeated for any value of the colon radius and any assumed position of the distal tip in relation to the center of the colon lumen. Although this method will reduce the computation time required to determine $\mathrm{X}_{\text {scope }}$ and $\mathrm{Y}_{\text {scope, }}$ it presents another problem. The purpose of this table is for the control system to search through it and find the values of $l_{1}, l_{2}$, and $l_{3}$ that most closely match the readings of the sensors. With a range of $0-30 \mathrm{~mm}$ for $\mathrm{X}_{\text {scope }}$ and $\mathrm{Y}_{\text {scope }}$, the size of the table is 29,578. This means that up to 88,734 operations will be needed to determine the position of the distal tip, which may take too long to keep up with the advancement of the colonoscope. However, due to the resolution of the sensors, the size of the table can be greatly reduced.

\section{Sensory system}

Figure 3 shows the sensory fixture mounted on the distal tip of the colonoscope. It has three sets of infrared sensors for the distance measurement between the distal tip and the colon wall. Each set of the infrared sensor has emitting and receiving parts and is placed at a $120^{\circ}$ interval to cover up the complete radial direction of the distal tip. The wires that can transmit the signal of the infrared sensors are through the biopsy channel in the colonoscope. The wires can be moved after the distal tip reaches the cecum, and the retraction procedure is the same as a conventional colonoscopy procedure.

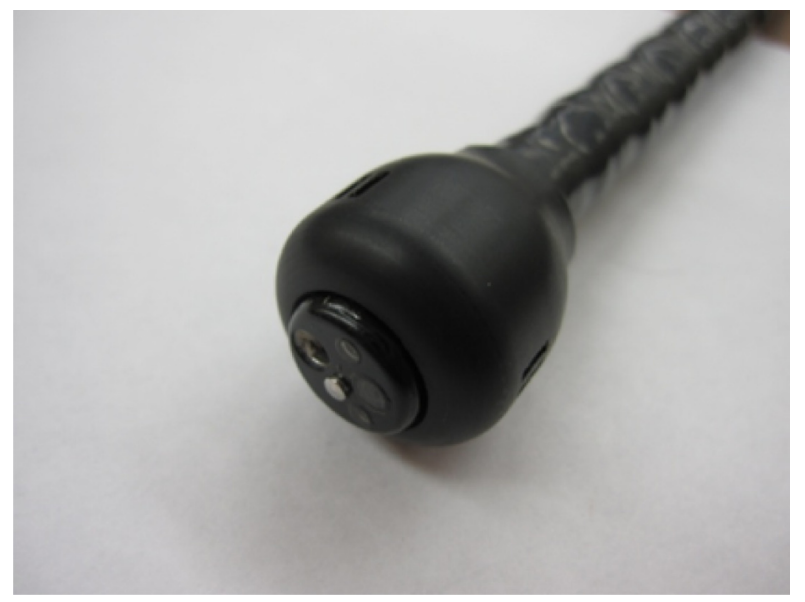

Figure 3 Sensory system of the colonoscopy add-on device.

The infrared sensors used in the system work by emitting and receiving infrared light. When the light hits the surface under examination, it is reflected back. The resulting output voltage is directly related to the distance from the sensor to the surface. In order to assess how much the voltage could vary, various surface conditions were tested. The output voltage from the infrared sensors in respect of the distances was measured and the results were analyzed. Secondly, the limited resolution of the infrared sensors was simulated and the possible solution was suggested to overcome the limitation of the hardware.

Sensor sensitivity was tested on lubricated and dry surface conditions. First, an infrared sensor was aimed at the surface that is normal to the sensor set. The voltage outputs were measured in respect of the distance to the test surface. The tests were on the silicon and polyvinylchloride surfaces, and the test results were compared.

The discrete, noncontinuous behavior of the sensor output limits the accuracy of the system in determining the position of the colonoscope tip. However, the purpose of the device is to avoid contact with the colon wall by approximately positioning the scope in the center of the colon lumen; therefore, it is acceptable if the position of the tip is within $5-10 \mathrm{~mm}$ of the actual center. Also, as mentioned previously, the resolution of the sensors presents the opportunity to significantly reduce the size of the look-up table for real-time control of the device. It was assumed that the values of $\mathrm{X}_{\text {scope }}$ and $\mathrm{Y}_{\text {scope }}$ could range anywhere from $0 \mathrm{~mm}$ to $30 \mathrm{~mm}$ in steps of $1 \mathrm{~mm}$. However, the sensors cannot differentiate between some distances (eg, 5-7 $\mathrm{mm}$ or $12-15 \mathrm{~mm}$ ). Averaging the range of possible distances to the colon wall for each sensor output value can describe the distance to within an accuracy of $2.5 \mathrm{~mm}$ (this was determined from the largest range of 
distances, $16-21 \mathrm{~mm}: 21-18.5=18.5-16=2.5 \mathrm{~mm})$. These averaged values can be used as the only possible values for $l_{1}, l_{2}$, and $l_{3}$. An algorithm can then be developed to pick only these discrete values from the look-up table and place them in another table. The size of the full table assuming a radius range of 0-30 mm resulted in a table of length 29,578 . Using the discrete table algorithm and assuming a threshold of $1 \mathrm{~mm}$, the size of the table can be reduced to 1735 . This means that the control system needs only to perform up to 5205 operations to find the position of the distal tip, which is a significant reduction of computation time.

\section{Actuation part}

The actuation part comprises two DC servo motors and the easily mountable or removable fixture. One DC servo motor is directly coupled to the dial knob of the colonoscope, and another one governs the dial knob through the timing belt and gears. The fixture is lightweight and easy to install or uninstall. Figure 4 shows the prototype of the actuation part mounted to the base of the colonoscope. In order to determine the actuator that can operate two dial knobs at the base of the colonoscope, control knob input torque was measured. Rotational speeds of two control knobs were experimentally determined for the optimal control of the distal tip of the colonoscope. Based on the knob rotations, the positions of the distal tip for both up-down and left-right movements were mapped using simple geometric calculation. The mapped information of the distal tip was also implemented through the control logic.

\section{Control system hardware}

The sensory and actuation parts are connected to a microprocessor and controlled by a programmed algorithm. The sensor

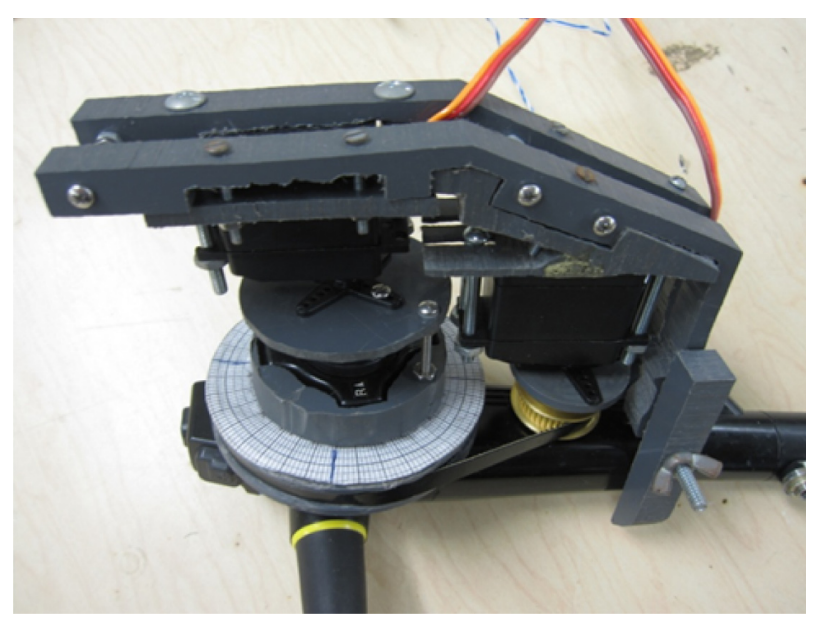

Figure 4 The prototype of the actuation part mounted to base of colonoscope. and motor drivers are imbedded in the control system, and the control system is connected to the sensory and actuation parts. External power supply is required for the system, and analog and digital powers are separated to minimize the possible noise to the system.

\section{Results}

Figure 5 shows the results of sensitivity tests. There was no difference in output voltage between the dry and lubricated surfaces when the sensor was uncovered. For every case, the voltage followed discrete steps for a range of distances. Once the distance of one range was exceeded, the voltage increased to another discrete value. The voltage increased faster in the silicone colon test; however, this increase is very small. The maximum variation in the distance for any voltage value is within $3 \mathrm{~mm}$. Therefore, the relationship between the output voltage of the sensors and the distance to the surface can be generalized, no matter what the test conditions are. Also, it can be noted that for every case, the voltage was identical up to $10 \mathrm{~mm}$. Furthermore, it can be assumed that the sensors will always have a protective covering and that the conditions inside the testing area will always be wet. In these conditions, for both the white and red test surfaces, the output voltage was identical up to $20 \mathrm{~mm}$.

\section{Effect of sensor resolution \\ on numerical tabulation}

Figure 6 shows a sample plot of the possible positions of the scope with the current sensor resolution. A colon radius of $10 \mathrm{~mm}$ was arbitrarily chosen for this example; in the actual table this value can range anywhere from $0 \mathrm{~mm}$ to $30 \mathrm{~mm}$. The blue stars represent all possible positions of the colonoscope tip for this colon radius. As is obvious from the plot, there are some "dead zones" shown in red; these are areas of the cross-section where the distal tip cannot be located due to the sensor resolution. It may seem that this empty space would cause the scope to be

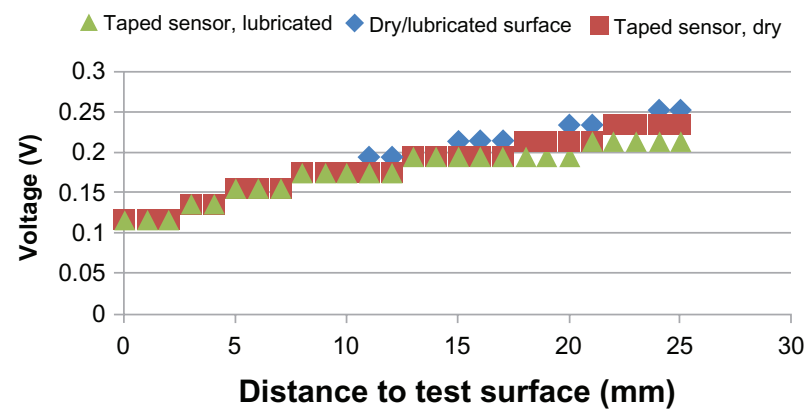

Figure $\mathbf{5}$ Sensor sensitivity test results on silicone colon. 


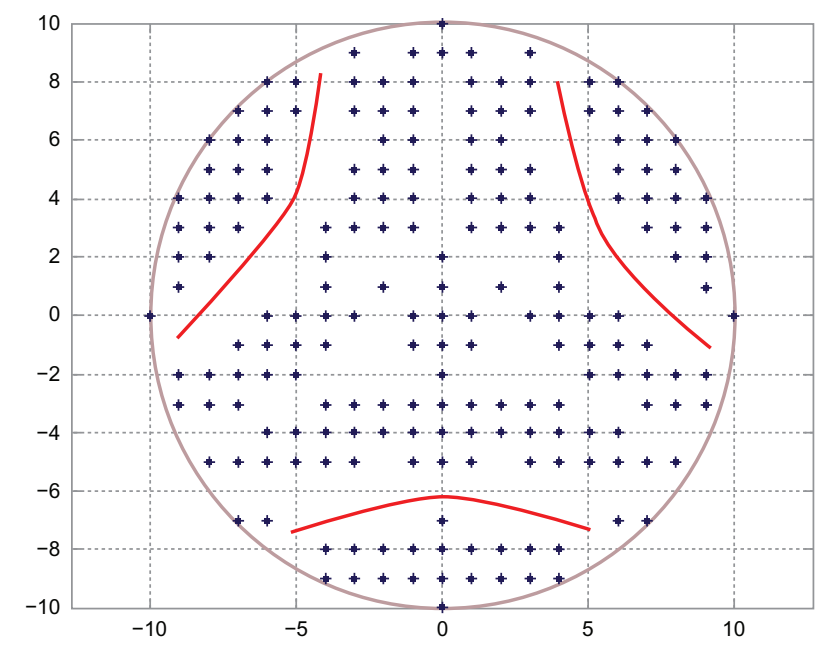

Figure 6 Sample grid of possible scope positions for $10 \mathrm{~mm}$ colon radius.

inaccurately moved to the center of the colon lumen; however, the device can act sequentially to achieve the correct motion.

Figure 7 shows the colonoscope tip at a "dead zone" position in the upper left quadrant of the colon crosssection. Point $S$ refers to the actual position of the scope, and points $a, b$, and $c$ show the possible positions that the control system calculates the scope can be. At points $a, b$, and $c$, the control system will move the scope according to line segments $a o, b o$, and $c o$. If these line segments are then shifted to the actual position of the tip, the scope will arrive at points $a^{\prime}, b^{\prime}$, and $c^{\prime}$. These points are very close to the center of the colon lumen. However, another movement can bring the tip exactly to the center. Figure 8 shows the second stage of the sequential movement. Points $a^{\prime}$ and $b^{\prime}$ are very close to possible tip positions as calculated in the discrete table. Consequently, the microprocessor will instruct the actuators to move the scope to almost the

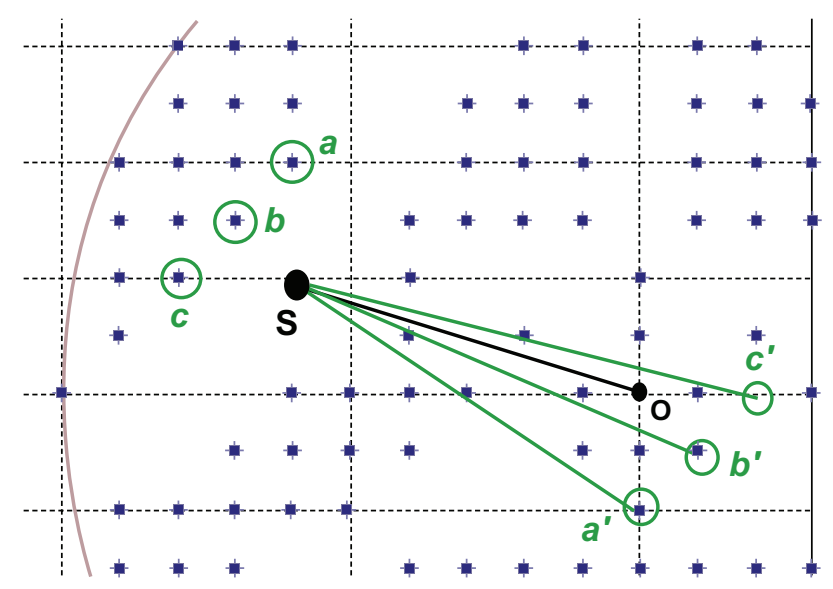

Figure 7 First stage of sequential movement when tip is in a "dead zone."

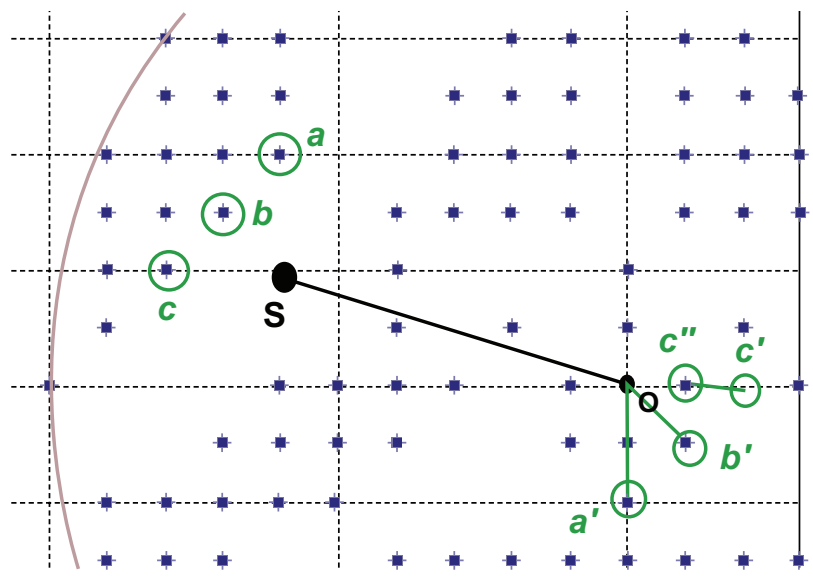

Figure 8 Second stage of sequential movement when the scope is in a "dead zone."

exact center of the colon lumen. Point $c^{\prime}$ is in between two discrete values; this will require a third sequential movement for proper positioning. This sequential positioning of the distal tip does not require additional programming; it will occur automatically with the previously mentioned control algorithm.

\section{Control knob input torque}

Figure 9 shows sample data for the input torque tests. As is seen in the graph, as the knob is rotated, the force increases linearly. After a small amount of rotation, the force drops suddenly. This drop in force does not affect the required input torque. After the drop, the force again begins to increase linearly until it reaches a maximum, which refers to the maximum rotation of the tip control knob. Once the knob is released, the force drops to zero.

\section{Determination of motor rotational speed}

Rotational grid paper was attached to the tip of the bottom dial. A reference point on the top dial was chosen to determine the amount of rotation of either knob. The microprocessor was programmed to rotate for a set amount of time and then

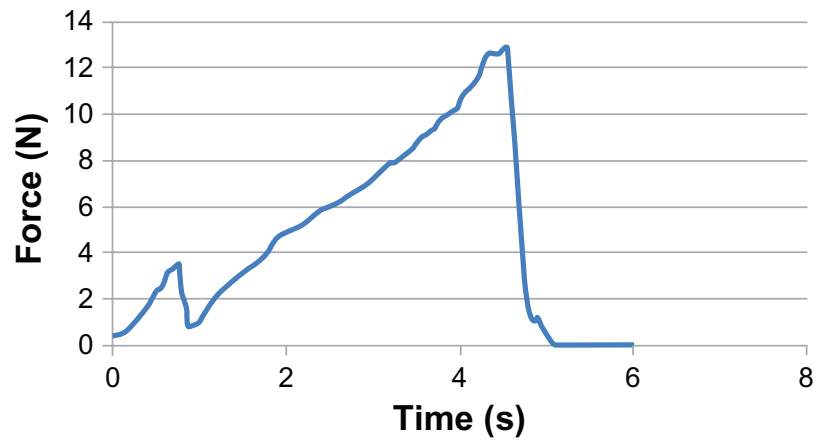

Figure 9 Sample data for input torque tests. 
stop, after which the amount of rotation was recorded. The amount of torque required to turn the knobs increases as the amount of knob rotation increases. Therefore, the rotational speed was tested at different starting positions of the knob rotation. Both knobs were tested at the maximum rotational speed of the servo motors. The results of this test are shown in Figures 10 and 11.

It can be seen that the top knob follows a smoother decrease in rotational speed as the starting position of the knob is increased than the bottom knob. The top knob is directly coupled to the DC servo motor and the control is more smooth and precise, whereas the bottom knob is connected with a timing belt and pulley. There is vibration and a slow response compared with the top knob. The different size of the top and bottom knobs is also the main reason for the slope difference between the two graphs.

\section{Mapping of control knob rotation to distal tip movement}

The position for the top control knob (left-right movement) and bottom control knob (up-down movement) as mapped is shown in Figures 12 and 13. Due to the mechanism, there are no movements from $5^{\circ}$ to $20^{\circ}$ rotation of the top and bottom knobs. Ten degrees of rotations of both knobs were approximately equal to each other.

As the tip of the knobs is rotated and the distal tip is moved, the angle in which the tip is pointing changes. As a consequence, to determine the amount that the scope must be moved, the angle must be taken into account. As is shown in Figures 12 and 13, the workspace of the distal tip follows a circular path. To determine the angle of the tip, the slope can be found using a central difference as in Equation (20):

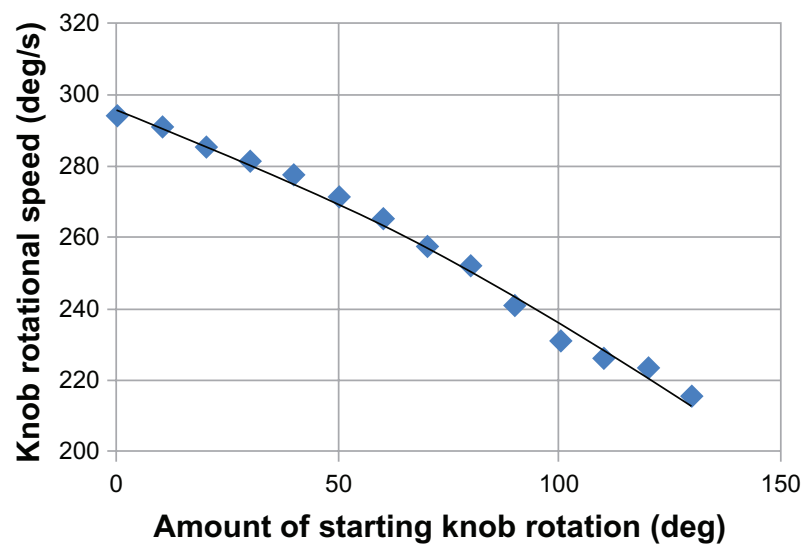

Figure 10 Top knob rotational speed.

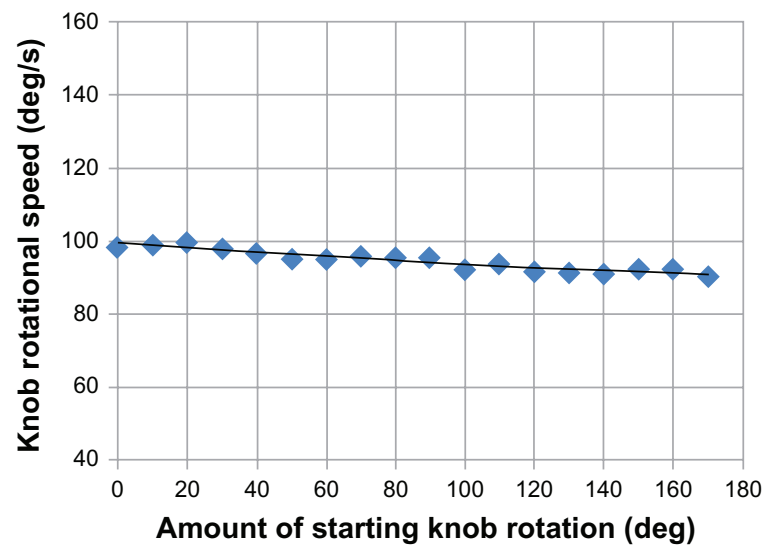

Figure I I Bottom knob rotational speed.

$$
\frac{d y_{i}}{d x_{i}}=\frac{y_{i+1}-y_{i-1}}{x_{i+1}-x_{i-1}}
$$

where $i$ is the current point. From this slope, trigonometry can be used to determine the angle of movement of the scope as in Equation (21):

$$
\theta_{i}=\tan ^{-1}\left(\frac{d y_{i}}{d x_{i}}\right)
$$

The values of the distal tip angle were calculated for every point. The largest difference in the angle for either knob is about $16^{\circ}$ at the maximum knob rotation. Generally, it will be uncommon for the scope to be moved to this far limit. During the knob rotation before the maximum, the difference in angle is, on average, $5.6^{\circ}$. If it is assumed that the maximum radius of the colon (and therefore the maximum movement necessary of the distal tip) is $30 \mathrm{~mm}$, the angle of the colonoscope would change by only $16.8^{\circ}$, on average.

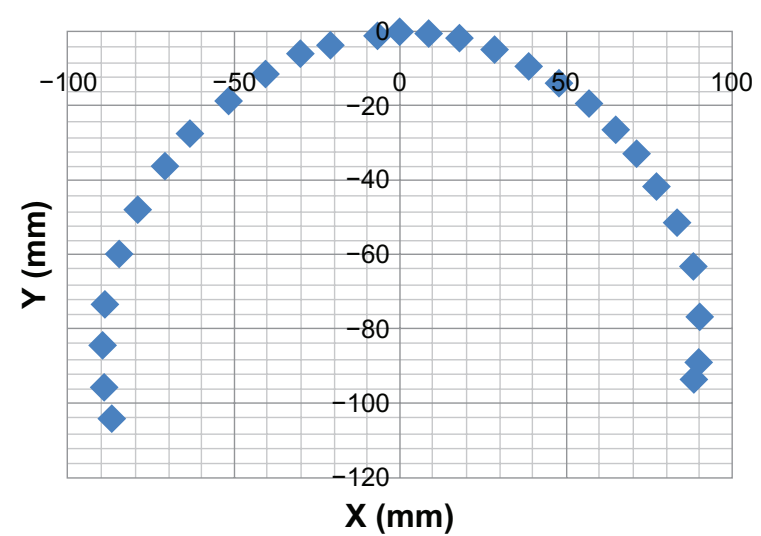

Figure 12 Tip position for top control knob (left-right movement). 


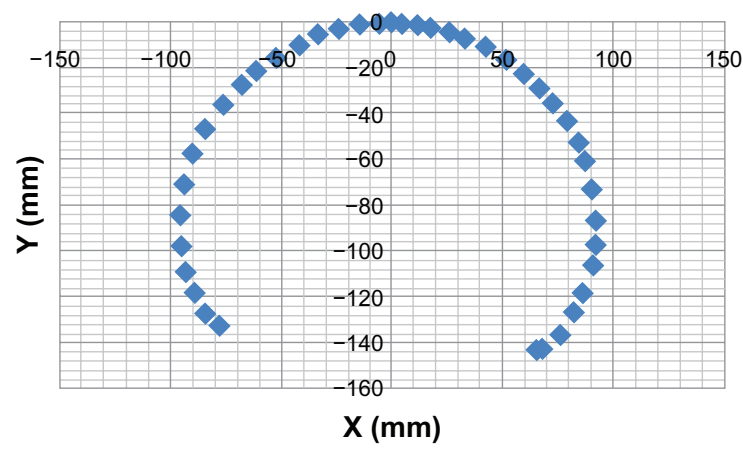

Figure 13 Tip position for bottom control knob (up-down movement).

Because this total angular movement is small, the small-angle approximation can be used. In this approximation, it can be assumed that the distal tip moves in a straight line when the tip is corrected back to the center of the colon lumen. The average distance for both clockwise and counterclockwise rotation for the bottom and top knobs was $10 \mathrm{~mm}$ and $11 \mathrm{~mm}$, respectively.

\section{Implementation of tip mapping in control logic}

The purpose of the sensory system is to pass the values of $\mathrm{X}_{\text {scope }}$ and $\mathrm{Y}_{\text {scope }}$, which are the $\mathrm{x}$ and $\mathrm{y}$ positions of the scope in relation to the center of the colon lumen, to the microprocessor. The next step in the operation of the automatically adjustable colonoscope is to utilize the actuators to move the distal tip to the center of the colon lumen - the procedure of this movement explained hereafter. It is assumed that when the colonoscopy is started, the distal tip is in the straight-out position, meaning that both of the tip control knobs have zero rotation, and the tip is starting in the center of the colon lumen. Once the tip deviates from the center, the sensory system passes the values of $\mathrm{X}_{\text {scope }}$ and $\mathrm{Y}_{\text {scope }}$ to the microprocessor. $X_{\text {scope }}$ refers to movement of the top knob, and $\mathrm{Y}_{\text {scope }}$ refers to movement of the bottom knob. The direction of the rotation of the knobs will be chosen based on the position of the distal tip. The rotational speed of the motors depends on the current amount of rotation of each knob. Therefore, the control system must always store the current amount of rotation. Table 1 shows the ranges of these values with the averaged rotational speed.

The relationship between the rotation of the tip control knobs and the distal tip movement was:

$$
\operatorname{map}_{B}=\frac{10^{\circ}}{10 \mathrm{~mm}}=1 \frac{\circ}{\mathrm{mm}} \text { and } \operatorname{map}_{T}=\frac{10^{\circ}}{11 \mathrm{~mm}}=0.91 \frac{\circ}{\mathrm{mm}}
$$

Table I Range of rotational speeds for bottom knob

\begin{tabular}{ll}
\hline Initial rotation $(\mathrm{deg})$ & $\omega(\mathrm{deg} / \mathbf{s})$ \\
\hline $0-30$ & 99.3 \\
$30-60$ & 96.9 \\
$60-90$ & 95.7 \\
$90-120$ & 94.1 \\
$120-150$ & 91.7 \\
$150-180$ & 92.0 \\
\hline
\end{tabular}

Using these relationships, the values of $X_{\text {scope }}$ and $\mathrm{Y}_{\text {scope }}$, and the motor rotational speed, Equation (26) can be developed to determine the amount of time each motor must be rotated to move the distal tip the desired amount:

$$
t_{T}=\frac{X_{\text {scope }} \text { map }_{T}}{\omega_{T}} \text { and } t_{B}=\frac{Y_{\text {scope }} \text { map }_{B}}{\omega_{B}}
$$

where $t$ is duration of rotation of each servo motor and $\omega$ is the rotational speed of the servo motors.

These equations do not fully describe the behavior of the servo motors. It was discovered that the first $0^{\circ}-30^{\circ}$ degrees of rotation of the tip control knobs resulted in no movement of the distal tip. This lag in the response must be incorporated into the control system to accurately and effectively position the colonoscope tip. Therefore, the control system must always monitor the current amount of rotation of the tip control knobs. Using this information, the control system can instruct the motors to rotate the knobs more than calculated, if necessary.

\section{Test of colonoscopy add-on device to colonoscopy training model}

For a test platform, the modified colonoscopy training model was used to validate the functionality of the colonoscopy add-on device. The colonoscopy training model comprised the human torso model, the rubber rings attached to the body, and a silicon colon.

Figure 14 shows the porcine colon in the colonoscopy training model. A silicon colon was replaced with a part of a porcine colon, and air was inserted from the cecum to inflate the porcine colon for realistic intubation. The porcine colon was restrained by ten rubber rings and firmly attached inside the colonoscopy training model. The total colonoscopy procedures were accomplished by the novice and expert. The intubation process took 14 minutes by the expert with the mucosa inspection, and it took 16 minutes by the novice without the mucosa inspection. The various parts of the porcine colon are captured in Figure 15. 


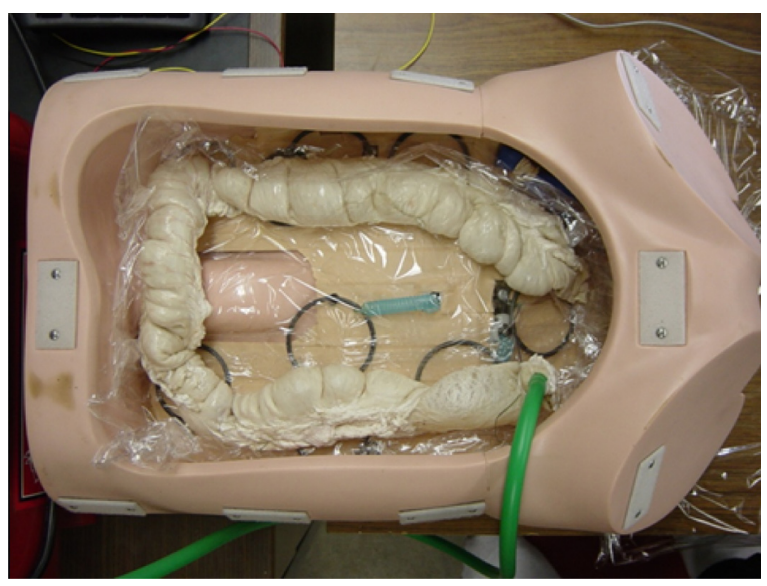

Figure 14 Colonoscopy training model with porcine colon. Air is inserted to open the lumen for more realistic intubation.

The distal tip of the colonoscope maintained its position in the lumen without contact with the colon wall. In some cases, it is unavoidable to make contact between the distal tip and the colon, and special cases during the intubation process are mentioned in the discussion section. After the distal tip reached the cecum, the colonoscopist removed the wiring connection through the biopsy channel and uninstalled the actuation part from the base of the colonoscope. The retreat process was the same as the normal colonoscopy procedure. The sensory fixture was still attached at the end of the distal tip, but it did not affect the motion of the distal tip, due to the low-profile design.

\section{Discussion}

The control theory assumes that the colonoscope is traveling through a straight, unobstructed passage of the colon. However, the actual human colon has several different sections with sharp bends. From the configuration of the sigmoid colon, it can be assumed that the control theory will be applicable during navigation of the descending colon,
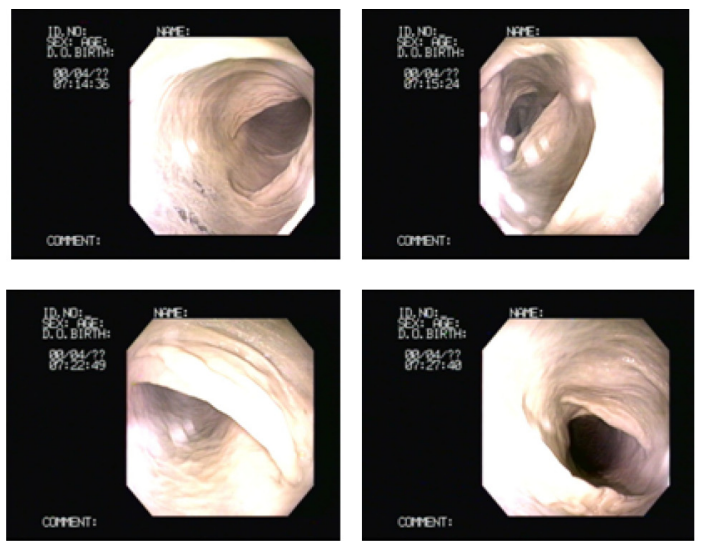
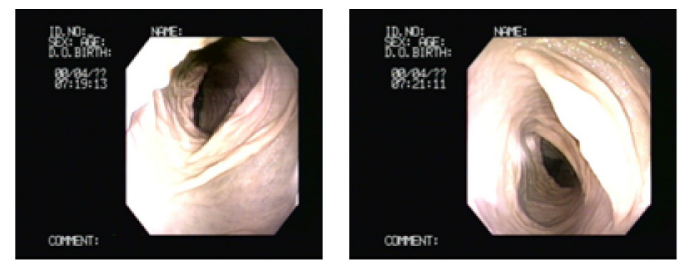

transverse colon, and ascending colon. However, there are four obvious places where the colon bends sharply: the first and second bends in the sigmoid colon, the bend in the left splenic flexure in between the descending and transverse colons, and the right hepatic flexure in between the transverse and ascending colons. As a result, it is necessary to add special cases to the programming of the microprocessor.

\section{Sigmoid colon}

Figure 14 shows the colon as if the patient were laying flat on their back. During colonoscopy, the patient is usually turned on their side. Therefore, it can be assumed that the sigmoid colon undergoes a bend of about $90^{\circ}$ upward. During this bend, the values of $l_{2}$ and $l_{3}$ will be consistent with normal sensor readings during navigation of straight passages of the colon. In other words, they will match some values in the look-up table. The value of $l_{1}$, however, will be greater than any values in the look-up table. As a result, the following steps can be taken to ensure proper navigation of the bend:

- Insert a condition in the programming of the microprocessor before it searches through the look-up table. This condition will be that $l_{2}$ and $l_{3}$ are less than the maximum sensor value available in the look-up table, whereas $l_{1}$ is greater than the maximum value.

- Instruct the servo motor connected to the up-down tip control knob to move the distal tip toward the direction of $l_{1}$

- Continue this movement until the value of $l_{1}$ is within the maximum sensor value.

Once these steps have been performed, the control system can resume normal operation of the device. Similarly, the second bend in the sigmoid colon can be navigated with different conditions. In this case, the value of $l_{1}$ will have a normal value found in the look-up table, whereas $l_{2}$ and

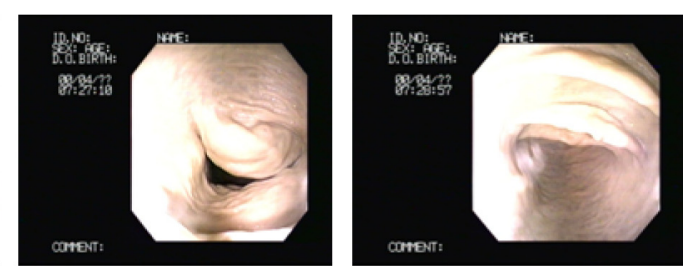

Figure 15 Snapshots of the porcine colon for the various parts during intubation. 
$l_{3}$ will have greater values. The up-down servo motor will then need to move the distal tip downwards to negotiate the bend. It should also be noted that every person's colon is differently shaped. Therefore, there may be other sections, not just in the sigmoid colon, that require this kind of movement. The bend correction algorithm mentioned previously can be extended to include all possible bends of this nature, utilizing readings from all sensors. The resulting motion will require the use of the up-down motor, the left-right motor, and a combination of both.

\section{Flattened colon, splenic flexure, and hepatic flexure}

The splenic flexure is located between the descending and transverse colons. The bend at this section is so sharp that often the colon lumen is completely flattened. As a result, the normal control logic will not apply to move the distal tip correctly. In this case, the bend correction logic mentioned previously will not work, because the bend direction will not be known, due to the flattened section. To navigate these sections, the control system can test several different movements to determine which direction the bend goes in. This can be accomplished by the following steps:

1. If all three sensors have very small readings $(<1 \mathrm{~mm})$, the microprocessor detects that the scope is entering a flattened section of the colon and will warn the colonoscopist with a beep or with a visual warning on the monitor.

2. The colonoscopist will then (slowly) continue to advance the scope. The microprocessor will continue to read the distance values from the sensor. If the sensor values return to normal values from the look-up table, it is determined that the flattened section is followed by a straight section of the colon, and normal operation of the device can resume.

3. If the sensor values do not return to normal, it is determined that the flattened section is followed by a bend. The control system will then instruct the actuators to bend the distal tip arbitrarily in one direction. If the sensor values return to normal, then it is determined that the bend has been negotiated successfully and normal operation of the device can resume.

4. If the sensor values do not return to normal, it is determined that the bend has not been negotiated successfully and the movement of the tip will be immediately stopped. Another warning signal will be issued to the colonoscopist to inform them to stop advancement of the scope and retract it.
5. The colonoscopist will repeat steps 3 and 4 . However, the microprocessor will instruct the actuators to move the scope in a different direction.

6. Steps $3-5$ will be repeated until it is determined that the scope has successfully negotiated the flattened section/ bend.

These flattened bends will occur at the splenic flexure, the hepatic flexure between the transverse colon and the ascending colon, and possibly between the rectum and the sigmoid colon. The algorithm described previously will be able to handle all of these possible scenarios.

The resolution of the sensors caused the device to not be able to discern between certain distances to the colon wall. This aspect of the device caused both advantages and disadvantages. It allowed the size of the look-up table to be reduced, reducing the amount of computation time required to determine the position of the scope and potentially decreasing the response time of the system. However, the low resolution of the sensors could cause problems in small-radius sections of the colon. If the distance sensed is incorrect by $2 \mathrm{~mm}$ in a part of the colon where the radius is $2 \mathrm{~mm}$, the scope could potentially come in contact with the colon wall. Another limitation was the effect of different types of light on the sensors. During the control tests in the colonoscopy training model, it was discovered that the halogen light emitted at the tip of the colonoscope caused erratic behavior of the sensors. The sensors would occasionally get "stuck" at some value (ie, the voltage would remain the same even when the sensor was moved away from the colon wall). This could be due to the glare of the light or possibly from the light being reflected off a circular surface, causing the light to be reflected unpredictably. The white light was changed to a green light, which solved the problem. However, the picture from the monitor is not as clear when the light is green, which could cause problems when attempting to diagnose abnormalities in the colon.

The tests performed on the actuation system provided an accurate description of the movement of the distal tip due to the rotation of the tip control knobs. During testing of the device, it was possible to achieve movement of the tip to the desired position. However, occasionally, the movement did not follow the predicted behavior of the tip mapping. This is due to the pull wire mechanism of the colonoscope. When the tip control knobs are rotated, they allow some wires to become tensioned while loosening others. This caused there to be some lag in the movement of the distal tip when the knobs were turned until the wires became fully tensioned. Although this did cause some inaccuracy in positioning, 
the device was able to position the distal tip approximately in the center of the colon lumen. The construction of the actuator fixture was robust enough to allow short-term use of the device without the need for maintenance.

\section{Conclusion}

A colonoscopy add-on device has been developed to improve the intubation process. A detachable sensory part measures the distance between the distal tip and the colon wall in three ways. A microprocessor integrates the signals from the sensory part and controls two dial knobs at the base of the colonoscope through an actuation system. It automatically indicates the direction of the lumen without direct contact with the colon wall, and intubation time is reduced. It also avoids the mechanical trauma caused by the end of the distal tip. After the distal tip reaches the cecum, the colonoscopist removes the wiring connected to the sensory fixture through the biopsy channel of the colonoscope and uninstalls the actuation part at the base of the colonoscope. The retraction process is the same as a conventional colonoscopy. The colonoscopy add-on device helps inexperienced colonoscopists to reduce intubation time and mechanical trauma, but there is a learning curve for an expert who is more familiar with a conventional colonoscope.

\section{Disclosure}

The authors report no conflicts of interest in this research.

\section{References}

1. Cotton PB, Williams CB. Practical gastrointestinal endoscopy. Oxford, UK: Blackwell Scientific Publications; 1990;1-7:214-215.

2. Hsieh YH, Zhou AL, Lin HJ. Long pediatric colonoscope versus intermediate length adult colonoscope for colonoscopy. J Gastroenterol Hepatol. 2008;23:e7-e10.

3. Ng WS, Phee SJ, Seow C, Davies BL. Development of a robotic colonoscope. Dig Endosc. 2000;12:131-135.

4. Aschoff AJ, Ernst AS, Brambs HJ, Juchems MS. CT colonography: an update. Eur Radiol. 2008;18:429-437.

5. Laghi A, Iannaccone R, Carbone I, et al. Detection of colorectal lesions with virtual computed tomographic colonography. Am J Surg. 2002;183:124-131.

6. Zijta FM, Bipat S, Stoker J. Magnetic resonance (MR) colonography in the detection of colorectal lesions: a systematic review of prospective studies. Eur Radiol. 2010;20:1031-1046.

7. Saar B, Meining A, Beer A, Settles M, Helmberger H, Frimberger E, et al. Prospective study on bright lumen magnetic resonance colonography in comparison with conventional colonoscopy. Br J Radiol. 2007;80: 235-241.

Medical Devices: Evidence and Research

\section{Publish your work in this journal}

Medical Devices: Evidence and Research is an international, peerreviewed, open access journal that focuses on the evidence, technology, research, and expert opinion supporting the use and application of medical devices in the diagnosis, treatment and management of clinical conditions and physiological processes. The identification of novel
8. Purkayastha S, Tekkis PP, Athanasiou T, et al. Magnetic resonance colonography versus colonoscopy as a diagnostic investigation for colorectal cancer: a meta-analysis. Clin Radiol. 2005;60: 980-989.

9. Pilz JB, Portmann S, Peter S, Beglinger C, Degen L. Colon capsule endoscopy compared to conventional colonoscopy under routine screening conditions. BMC Gastroenterol. 2010;10(66):1-7.

10. Sacher-Huvelin S, Coron E, Gaudric M, Planche L, Benamouzig R, Maunoury V. Colon capsule endoscopy vs colonoscopy in patients at average or increased risk of colorectal cancer. Aliment Pharmacol Ther. 2010;32:1145-1153.

11. Gossum AV, Navas MM, Fernandez-Urien I, Carretero C, Gay G, Delvaux M, et al. Capsule endoscopy versus colonoscopy for the detection of polyps and cancer. N Engl J Med. 2009;361(3):264-270.

12. Brooker JC, Saunders BP, Shah SG. A new variable stiffness colonoscope makes colonoscopy easier: a randomised controlled study. Gut. 2000;46:801-805.

13. Chen PJ, Shih YL, Chu HC, Chang WK, Hsieh TY, Chao YC. A prospective trial of variable stiffness colonoscopes with different tip diameters in unsedated patients. Am J Gastroenterol. 2008;103: $1365-1371$.

14. Kaffes AJ, Mishra A, Ding SL, et al. A prospective trial of variable stiffness pediatric vs standard instrument colonoscopy. Gastrointest Endosc. 2003;58(5):685-689.

15. Othman MO, Bradley AG, Choudhary A, Hoffman RM, Roy PK. Variable stiffness colonoscope versus regular adult colonoscope: metaanalysis of randomized controlled trials. Endoscopy. 2009;41:17-24.

16. Horiuchi A, Nakayama Y, Kajiyama M, Fujii H, Tanaka N. Usefulness of a small-caliber, variable stiffness colonoscope as a backup in patients with difficult or incomplete colonoscopy. Am J Gastroenterol. 2004;99: 1936-1940.

17. Raju GS, Rex DK, Kozarek RA, Ahmed I, Brining D, Pasricha PJ. A novel shape-locking guide for prevention of sigmoid looping during colonoscopy. Gastrointest Endosc. 2004;59(3):416-419.

18. Yagi A, Matsumiya K, Masamune K, Liao H, Dohi T. Rigid-flexible outer sheath model using slider linkage locking mechanism and air pressure for endoscopic surgery. Med Image Comput Comput Assist Interv. 2006;4190:503-510.

19. Loeve AJ, Van de Ven OS, Vogel JG, Breedveld P, Dankelman J. Vacuum packed particles as flexible endoscope guides with controllable rigidity. Granular Matter. 2010;12:543-554.

20. Eickhoff A, Van Dam J, Jakobs R, Kudis V, Hartmann D, Damian U, et al. Computer-assisted colonoscopy (the NeoGuide Endoscopy System): results of the first human clinical trial ("PACE Study"). Am J Gastroenterol. 2007;102:261-266.

21. Kumar S, Kassim IM, Asari VK. Design of a vision-guided microrobotic colonoscopy system. Adv Robot. 1999;14(2):87-104.

22. Zuo J, Yan G, Gao Z. A micro creeping robot for colonoscopy based on the earthworm. J Med Eng Technol. 2005;29(1):1-7.

23. Ye D, Yan G, Wang K, Ma G. Development of a micro-robot for endoscopes based on wireless power transfer. Minim Invasive Ther. 2008; 17(3):181-189.

24. Litten J, Choi J. Development of an automatically adjustable colonoscope. DMD 2011 - Proceedings of the 2011 Design of Medical Device Conference, April 12-14, 2011, Minneapolis, MN.

\section{Dovepress}

devices and optimal use of existing devices which will lead to improved clinical outcomes and more effective patient management and safety is a key feature. The manuscript management system is completely online and includes a quick and fair peer-review system. Visit http://www. dovepress.com/testimonials.php to read real quotes from authors. 